\title{
ALILBORACION DEL BENZALDEHIDO CATALIZADA CON ACIDO DE LEWIS. UN ESTUDIO DE LA DENSIDAD DE CARGA ELECTRONI- CA Y SU LAPLACIANO
}

\author{
Margarita M. Vallejos(1), María V. Avanza(1), María G. Chaves ${ }^{(2)}$ y Nélida M. Peruchena ${ }^{(1)}$
}

RESUMTN: En este trabajo se realiza un estudio basado en la teoría de átomos en moléculas sobre distintas especies químicas involucradas en el mecanismo de reacción propuesto para el alilboronato de pinacol (1) con benzaldehído (2) y una molécula de $\mathrm{AlCl}_{3}$, como catalizador ácido de Lewis. Diferentes propiedades topológicas de la densidad de carga electrónica $(\rho)$ y su Laplaciano $\left(\nabla^{2} \rho\right)$ son evaluadas como potenciales indicadores de reactividad en la alilboración. Los resultados muestran que la coordinación entre $\mathrm{AlCl}_{3}$ y el reactivo (1 ó 2) conlleva a una particular distribución de la densidad electrónica en los complejos reactivo-catalizador y en las estructuras de transición, la cual puede ser evaluada a través de diferentes parámetros de la topología de $\rho$ en los puntos críticos de enlace seleccionados. El análisis topológico de $\nabla^{2} \rho$ en los complejos reactivo-catalizador brinda una valiosa información sobre los sititos más susceptibles al ataque nucleofílico y electrofílico involucrados en la reacción bajo estudio.

ABSTR $\wedge$ CT: In this work a study on the chemical species involved in the proposed reaction mechanism of pinacol allylboronate (1) with benzaldehyde(2) and $\mathrm{AlCl}_{3}$ molecule as a Lewis acid catalyst is carried out within the framework of the atom in molecule theory. Several topological properties of the electron density $(\rho)$ and its Laplacian $\left(\nabla^{2} \rho\right)$ were investigated as potential indicators of reactivity of allylboration. The results show that the coordination of $\mathrm{AlCl}_{3}$ molecule to the reactant (1 or 2$)$ lead to a particular electron charge distribution on the reactant-catalyst complexes and the transition structures, which can be evaluated by different topological properties of $\rho$ at the selected bond critical point. The topological analysis of $\nabla^{2} \rho$ at the reactive-catalyst complexes provides valuable information about the most susceptible sites to nucleophilic and electrophilic attack involved in the reaction under study.

Palabras claves: compuestos organoboro, QTAIM, DFT

Key words: organoboron compounds, QTAIM, DFT

\section{INTRODUCCIÓN}

La adición de un reactivo organometálico alílico a un compuesto carbonílico es una poderosa herramienta en síntesis orgánica con la cual además de formarse enlaces C$\mathrm{C}$, se genera un nuevo grupo funcional y se origina un nuevo estereocentro (Hoffmann, 1982, Yamamoto et al., 1993; Denmark et al., 2003; Kennedy et al., 2003). La reacción de alilación de compuestos aliliboro es extremamente útil debido a que se obtienen productos con excelente diasteroselectividad (Hoffmann et al., 1981, Hoffmann, 1982). E1 origen de la diasteroselectividad que muestran estas reacciones fue explicada mediante la

1) Facultad de Ciencias Exactas y Naturales y Agrimensura. Universidad Nacional del Nordeste (UNNE) and Consejo Nacional de Investigaciones Científicas y Técnicas (CONICET). Av. Libertad 5470 (3400) Corrientes, Argentina.

E-mails: vallejos.marga@gmail.com; vavanza@yahoo.es; arabeshai@yahoo.com.ar

2) Facultad de Ciencias Exactas y Naturales y Agrimensura. Universidad Nacional del Nordeste (UNNE). Av. Libertad 5450 (3400) Corrientes, Argentina. E-mail: chavesgm2@gmail.com 
formacion de una estructura de transición (ET) cíclica de seis miembros en la cual se produce la activación interna del carbonilo por el átomo de boro (Hoffmann et al., 1979; Corey et al., 1989; Li et al.,1989; Gung et al., 2002).

Brown y colaboradores (Brown et al., 1990) investigaron el efecto del solvente, la temperatura y la estructura de los compuestos alilboro sobre la velocidad de la reacción de alilboración y sugirieron que la reactividad de los compuestos alilboro estaba relacionada con la electrofilicidad del átomo de boro. Asimismo, estudios teóricos mostraron una buena correlación entre la electrofilicidad estimada del átomo de boro y la energía de activación calculadas por métodos ab initio (Omoto et al., 1998).

Miyaura y colaboradores (Ishiyama et al., 2002) reportaron que la reacción del alilboronato de pinacol con aldehídos realizada a $-78^{\circ} \mathrm{C}$ es acelerada por los ácidos de Lewis como $\mathrm{AlCl}_{3}$ y $\mathrm{Sc}(\mathrm{OTf})_{3}$, mientras que la reacción no transcurre en ausencia de ácido de Lewis. A partir de la alilboración catalítica estos autores lograron obtener alcoholes homoalílicos con excelente diastereoselectividad pero moderado rendimiento y enantioselectividad.

Para comprender el rol del ácido de Lewis como catalizador en las reacciones de alilboración Sakatay Fujimoto(Sakata et al., 2008) realizaron un estudio teórico de la reacción entre el alilboronato de pinacol y el benzaldehído en ausencia y en presencia de $\mathrm{AlCl}_{3}$ (en su forma monomérica). Estos autores investigaron diferentes caminos de reacción dependiendo del sitio de unión entre el $\mathrm{AlCl}_{3}$ y uno de los oxígenos del reactivo (los oxígenos del pinacol y del carbonilo). La reacción de alilboración sin catalizador tiene una barrera energética relativamente alta, mientras que el camino de reacción que involucra una ET con el $\mathrm{AlCl}_{3}$ coordinado a uno de los oxígenos del boronato de pinacol presenta una barrera energética significativamente baja. Mediante el análisis de población electrónica y de orbitales moleculares se encontró que la interacción entre el $\mathrm{AlCl}_{3}$ y un oxígeno del boronato mejora la electrofilicidad del átomo de boro y a su vez, disminuye la nucleofilicidad del doble enlace $\mathrm{C}=\mathrm{C}$ de alilboronato. Estos resultados obtenidos mediantes cálculos teóricos, concuerdan con el mecanismo de activación nucleofílica de boronatos propuesto previamente a partir de estudios experimentales y cinéticos. (Kennedy et al., 2002; Kennedy y Hall, 2003; Lachance et al., 2003; Kennedy et al., 2004; Rauniyar et al.,2004).

La teoría cuántica de átomos en moléculas (QTAIM del inglés the quantum theory of atom in molecules) (Bader, 1990; Matta et al., 2007b) basada en los principios de la mecánica cuántica, permite conocer la estructura electrónica de una molécula, y la naturaleza y propiedades de un enlace químico (Bader, 1990; Matta y Boyd, 2007b). En base a esta teoría, una reacción química corresponde a la combinación de una región de concentración de carga de una base con una región de disminución de carga de un ácido en la capa de valencia de concentración de carga. El análisis topológico de la densidad electrónica y su Laplaciano fue utilizado exitosamente para racionalizar el mecanismo de diversas reacciones químicas como la dimerización del ciclopentadieno (Werstiuk et al., 2008), el reordenamiento de Cope del 1,5-hexadieno (Brown et al., 2009), las reacciones pericíclicas y pseudopericíclicas (Rode et al., 2007; Calvo-Losada et al., 2008, López et al., 2011 ) entre otras (Wagner et al., 2007; Zalazar et al., 2011; Zalazar et al., 2013). Nosotros hemos realizados estudios en el marco de la QTAIM sobre las reacciones de 
Diels-Alder de compuestos alquenil y alquinilboro (Vallejos et al., 2013; Vallejos et al., 2014 b; Vallejos et al., 2015) y encontramos que la reactividad relativa de dienofilos activados con boro puede ser estimada mediante diversos parámetros topológicos dentro de la topológica del Laplaciano de la densidad de carga electrónica evaluadas sobre la moléculas de los reactivos (Vallejos et al., 2014b).Como las reacciones de alilboración están muy relacionadas con las reacciones de Diels-Alder de compuestos organoboro, se pretende extender el estudio de los parámetros topológicos de la densidad electrónica y su Laplaciano a las reacciones de alilboración, para lograr un mayor entendimiento de la reactividad de las especies químicas involucradas en el mecanismo de esta interesante reacción.

En este trabajo se realiza un análisis topológico de la densidad de carga electrónica y su Laplaciano en el contexto de la teoría QTAIM sobre las especies químicas involucradas en la reacción entre el alilboronato de pinacol (1) y el benzaldehído (2) en presencia y ausencia de $\mathrm{AlCl}_{3}$ como catalizador (Esquema 1), con el objeto de obtener distintos parámetros que permitan evaluar el efecto del catalizador sobre la electrofilicidad y nucleofilicidad de los reactivos y sobre la distribución de carga electrónica de las ETs implicadas en la alilboración.

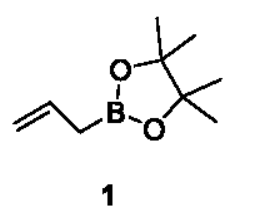

1

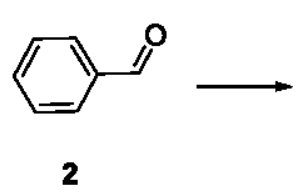

Esquema 1

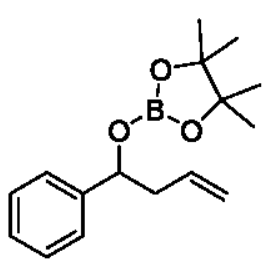

\section{METODOLOGÍA}

Las geometrías de las especies químicas involucradas en los diferentes caminos de la reacción entre 1 y 2 con y sin catalizador obtenidas a nivel B3LYP/6-311G(d,p), por Sakata y Fujimoto (Sakata and Fujimoto, 2008) fueron utilizadas en el presente estudio como estructuras de partida. Las geometrías de los reactivos, complejos de reacción, ETs y productos fueron optimizadas utilizando el funcional global hibrido Meta-GGA MPWB1K (Zhao et al., 2004) con el conjunto de bases 6-311G (d,p). Varios autores (Domingo et al., 1998; Domingo et al., 2013; Domingo et al., 2014; Vallejos et al., 2014a; Nacereddine et al., 2015) informaron que los cálculos realizados con el funcional MPWB1K arrojan valores termodinámicos relativamente exactos, mientras que con el funcional B3LYP se subestiman las energías de reacción (Check et al., 2005; Griffith et al., 2006; Jones et al., 2006). Las energías libre fueron calculadas al mismo nivel que la optimización geométrica, a 298,15 K y 1 atm. Se calcularon las frecuencias para caracterizar las estructuras optimizadas como mínimos energéticos sobre la superficie de energía potencial (ausencia de frecuencias imaginarias) o como estructuras de transición (una 
frecuencia imaginaria). Los cálculos fueron realizados con el programa Gausssian 09 (Frisch, 2009).

Las propiedades topológicas locales de la densidad electrónica y su Laplaciano y las propiedades atómicas integradas para las especies químicas seleccionadas, se obtuvieron con el programa AIMALL (Keith, 2011). Para evaluar la exactitud de los cálculos de integración se consideró valores del Laplaciano de la densidad electrónica (en valor absoluto), $\left|L_{\mathrm{r}}\right|$, menores a $10^{-5}$ ua. para los átomos de hidrógeno y menores a $4 \times 10^{-4} \mathrm{ua}$. para los demás átomos.(Blieger-Konig, 2000).

\section{RESULTADOS Y DISCUSIÓN}

Sakata y colaboradores (Sakata y Fujimoto, 2008) analizaron cuatro caminos para la reacción entre 1 y $\mathbf{2}$, con y sin catalizador. $\mathrm{El}$ camino A corresponde a la reacción en ausencia de catalizador, en los caminos $\mathrm{B}$ y $\mathrm{C}$ el $\mathrm{AlCl}_{3}$ interactúa con uno de los oxígenos del boronato de 1 y en el camino $\mathrm{D}$ el $\mathrm{AlCl}_{3}$ interactúa con el oxígeno carbonílico de 2. En los caminos B, C y D primero se forman complejos reactivo-catalizador, $\mathbf{C C},(\mathbf{C C}$ $\mathbf{B}, \mathbf{C C}-\mathbf{C}$ y CC-D). En los cuatros caminos se forman complejos de reacción $\mathbf{C R}$, entre $\mathbf{1}$ y $\mathbf{2}$ en el camino A (CR-A), entre los complejos CC-B y CC-C y 2, (CR-B y CR-C) en los caminos B y C, respectivamente y entre CC-D y 1 (CR-D) en el camino D. La ET de cada camino, ET-A, ET-B, ET-C y ET-D conduce a los correspondientes productos PA, P-B, P-C y P-D respectivamente. Estos autores analizaron y discutieron los parámetros geométricos y energéticos de las especies mencionadas, por lo tanto, en este trabajo nos focalizaremos en el análisis de la distribución de la densidad de carga electrónica en los reactivos y los cambios que ocurren debido a la interacción con el catalizador en los CC, y en las ETs. En la Tabla 1 se exponen las energías libre de Gibbs relativas de todas las especies químicas que interviene en los cuatro caminos de reacción, junto con las energías reportada por Sakata y colaboradores.

Nosotros reexaminamos los parámetros geométricos y energéticos usando el nivel de teoría MPWB1 K/6-311G(d,p), encontrando que las geometrías no muestran cambios significativos respecto a las obtenidas con el funcional B3LYP por Sakata y colaboradores. En general, las energías calculadas con el funcional MPWB1K son más bajas que las calculadas con B3LYP, pero muestran la misma tendencia. Las energías libre de Gibbs relativas calculadas a $298,15 \mathrm{~K}$ para las ET-B y ET-C son considerablemente bajas y de signo negativo a diferencias de las calculadas con el funcional B3LYP, indicando que no existe barrera energética (Domingo et al., 2002). Sin embargo, como las reacciones de alilboración fueron realizadas a $195,15 \mathrm{~K}\left(-78^{\circ} \mathrm{C}\right)$, estos resultados indican que la presencia del $\mathrm{AlCl}_{3}$ mejora la velocidad de reacción en concordancia con los resultados experimentales (Kennedy y Hall, 2002). 
Tabla 1: Energía libre de Gibbs relativa ( $\Delta G$, en $\mathrm{kcal} / \mathrm{mol}$ ) calculadas con los funcionales $\mathrm{MPWB} 1 \mathrm{~K}$ y B3LYP con el conjunto base 6-311G(d,p)."

\begin{tabular}{lcc}
\hline Especies & $\Delta G_{\text {YPWB }}$ & $\Delta G_{B 34 Y p^{\mathrm{h}}}{ }^{\mathrm{n}}$ \\
\hline CR-A & 8,5 & 7,4 \\
ET-A & 21,2 & 30,1 \\
P-A & $-20,5$ & $-13,3$ \\
\hline CC-B+2 & $-18,5$ & $-11,2$ \\
CR-B & $-18,8$ & $-2,3$ \\
ET-B & $-12,4$ & 6,3 \\
P-B & $-43,0$ & $-27,0$ \\
\hline CC-C+2 & $-18,3$ & $-10,0$ \\
CR-C & $-10,5$ & 5,5 \\
ET-C & $-6,0$ & 12,5 \\
P-C & $-38,1$ & $-22,6$ \\
\hline 1+CC-D & $-21,7$ & $-17,1$ \\
CR-D & $-12,9$ & $-11,2$ \\
ET-D & 5,55 & 23,8 \\
P-D & $-36,1$ & $-20,0$ \\
\hline
\end{tabular}

"Las energías de las especies asociadas con el camino de reacción A son relativas a la suma de las energías de $1+2$ y las de los caminos B, C. y D son relativas a la suma de las energías de $1+2+\mathrm{AlCl}_{3}$. ${ }^{\text {b }}$ Datos energéticos calculados a nivel B3LYP/6-311G(d,p) reportados por Sakata y colaboradores.

\section{Análisis topológico de la densidad electrónica}

Los fundamentos del análisis topológico de la densidad electrónica en el marco de QTAIM (Bader, 1990; Matta y Boyd, 2007b) están bien documentados en la literatura, por lo tanto en el presente trabajo se brindará una breve reseña de los aspectos esenciales para la discusión.

Varios paramentos topológicos evaluados en los puntos críticos de enlace (pce) (con el subíndice "b" se identifican las propiedades en el pce) pueden ser utilizados para obtener información de la naturaleza y características de un enlace químico. La densidad electrónica $\left(\rho_{\mathrm{b}}\right)$ en un pce refleja la fortaleza del enlace, y su Laplaciano $\left(\nabla^{2} \rho_{\mathrm{b}}\right)$ indica las regiones de concentración $\left(\nabla^{2} \rho_{\mathrm{b}}<0\right)$ o disminución de carga local $\left(\nabla^{2} \rho_{\mathrm{b}}>0\right)$. Estos dos parámetros junto con la relación entre la densidad de energía potencial, en valor absoluto, y la densidad de energía cinética $\left(\left|V_{\mathrm{h}}\right| / G_{\mathrm{b}}\right)$ son utilizados para analizar el carácter covalente de una interacción.

Dos tipos de interacciones pueden distinguirse mediante los parámetros topológicos, las interacciones de capa compartida que corresponden a los enlaces covalente y generalmente presentan valores relativamente altos de $\rho_{\mathrm{b}}$ y $\nabla^{2} \rho_{\mathrm{b}}<0$, y las interacciones de capa cerrada $\rho_{\mathrm{b}}$ que muestran valores de $\rho_{\mathrm{b}}$ relativamente bajos, $\nabla^{2} \rho(\mathrm{r})>0$ y la contribución de la energía cinética es mayor que la contribución de la energía potencial $\left(\left|V_{\mathrm{h}}\right| / G_{\mathrm{b}}<1\right)$. 


\section{Reactivos y complejos reactivos-catalizador $(\boldsymbol{C C})$}

En la Fig. 1 se muestran los grafos moleculares de los reactivos $\mathbf{1}$ y $\mathbf{2}$ y $\operatorname{los}$ complejos CC-B, CC-C y CC-D. En la Tabla 1 se expresan las distancias de enlaces y las propiedades topológicas seleccionadas sobre los pces más relevantes para la discusión.

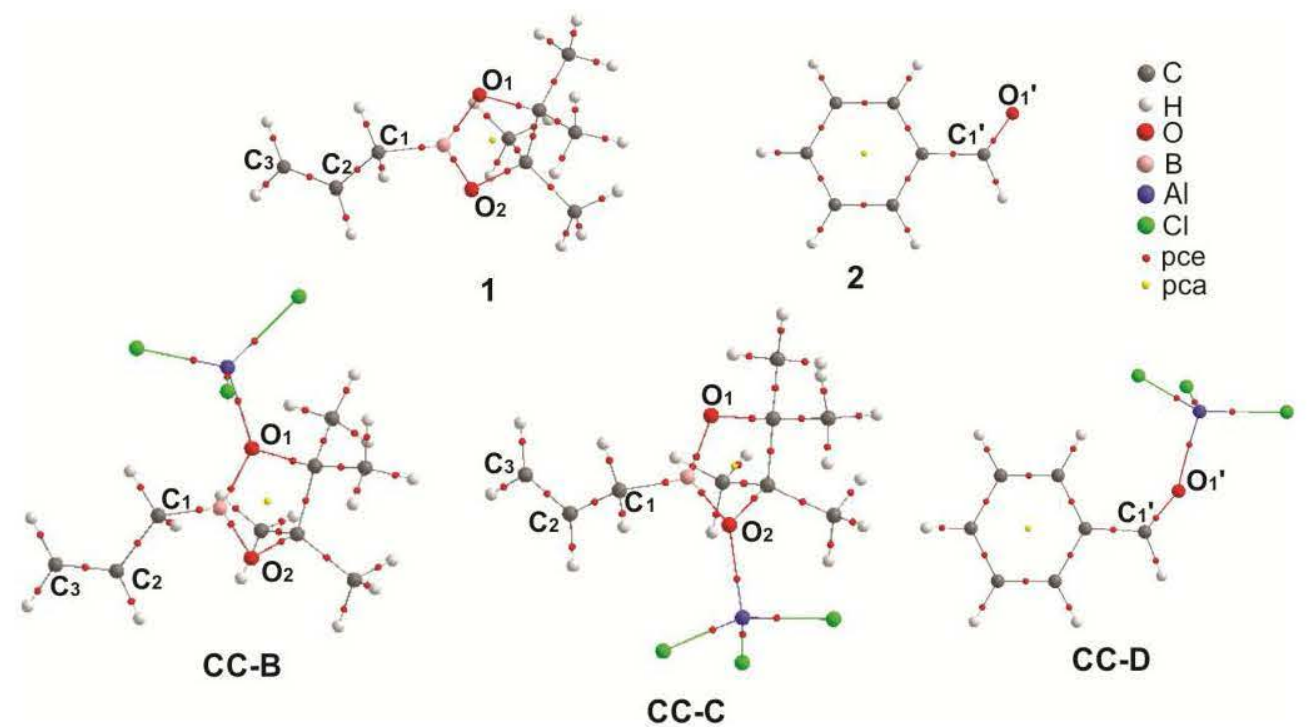

Fig. 1: Grafos moleculares de los reactivos 1 y 2 y de los complejos reactivo-catalizador CC-B. CC-C y CC-D.

Tabla 2: Distancias de enlace y propiedades topológicas locales en los pces de los enlaces seleccionados en los reactivos y complejo reactivo-catalizador bajo estudio. ${ }^{2}$

\begin{tabular}{|c|c|c|c|c|c|}
\hline Especie & interacción & $d$ & $\rho_{b}$ & $\nabla^{2} \rho_{b}$ & $\left|V_{\mathrm{b}}\right| / G_{\mathrm{b}}$ \\
\hline \multirow{3}{*}{1} & B-C ${ }_{1}$ & 1,56 & 0,189 & $-0,254$ & 2,467 \\
\hline & $\mathrm{B}-\mathrm{O}_{1}$ & 1,36 & 0,206 & 0,983 & 1,399 \\
\hline & $\mathrm{B}-\mathrm{O}_{2}$ & 1,36 & 0,205 & 0,977 & 1,400 \\
\hline 2 & $\mathrm{C}_{1}{ }^{\prime}-\mathrm{O}_{1}{ }^{\prime}$ & 1,19 & 0,419 & 0,241 & 1,922 \\
\hline \multirow{4}{*}{ CC-B } & $\mathrm{Al} \cdots \mathrm{O}_{1}$ & 1,92 & 0,055 & 0,392 & 0,932 \\
\hline & B-C ${ }_{1}$ & 1,55 & 0,197 & $-0,347$ & 2,683 \\
\hline & $\mathrm{B}-\mathrm{O}_{1}$ & 1,42 & 0,168 & 0,807 & 1,366 \\
\hline & $\mathrm{B}-\mathrm{O}_{2}$ & 1,38 & 0,219 & 1,042 & 1,411 \\
\hline \multirow{4}{*}{$\mathrm{CC}-\mathrm{C}$} & $\mathrm{Al} \cdots \mathrm{O}_{2}$ & 1,91 & 0,056 & 0,406 & 0,928 \\
\hline & $\mathrm{B}-\mathrm{C}_{1}$ & 1,55 & 0,197 & $-0,341$ & 2,666 \\
\hline & $\mathrm{B}-\mathrm{O}_{1}$ & 1,34 & 0,219 & 1,048 & 1,410 \\
\hline & $\mathrm{B}-\mathrm{O}_{2}$ & 1,43 & 0,166 & 0,787 & 1,365 \\
\hline \multirow[t]{2}{*}{ CC-D } & $\mathrm{Al} \cdots \mathrm{O}_{1}{ }^{\prime}$ & 1,87 & 0,057 & 0,452 & 0,904 \\
\hline & $\mathrm{C}_{1}{ }^{\prime}-\mathrm{O}_{1}{ }^{\prime}$ & 1,22 & 0,378 & 0,270 & 1,900 \\
\hline
\end{tabular}

${ }^{2}$ Las distancias de enlace $(d)$ se expresan en $\AA$ y los valores de $\rho_{\mathrm{b}}$ y $\nabla^{2} \rho_{\mathrm{b}}$ en unidades atómicas (ua.). $\left|V_{b}\right| / G_{b}$ es adimensional. 
En los grafos moleculares de CC-B y CC-C se observan los caminos de enlaces $\mathrm{O}_{1} \cdots \mathrm{Al}$ y $\mathrm{O}_{2} \cdots \mathrm{Al}$, respectivamente correspondientes a la interacción entre $\mathrm{AlCl}_{3}$ y uno de los oxígenos del boronato de 1 . Ambas interacciones muestran características de interacciones de capa cerrada, valores relativamente bajos $\rho_{\mathrm{b}}, \nabla^{2} \rho_{\mathrm{b}}>0$, y $\left|V_{\mathrm{b}}\right| / G_{\mathrm{b}}<1$. La distancia de enlace y $\rho_{\mathrm{b}}$ indican que la interacción $\mathrm{O}_{1} \cdots \mathrm{Al}\left(d=1,92 \AA, \rho_{\mathrm{b}}=0,055\right.$ ua.) es ligeramente más débil que $\mathrm{O}_{2} \cdots \mathrm{Al}\left(d=1,91 \AA, \rho_{\mathrm{b}}=0,056\right.$ ua.), en concordancia con la energía calculada para ambos compuestos.

En comparación con el reactivo 1, los enlaces $\mathbf{B}-\mathrm{O}_{1}$ y $\mathrm{B}-\mathrm{O}_{2}$ en $\mathbf{C C}-\mathbf{B}$ y $\mathbf{C C}-\mathbf{C}$, respectivamente se alargan $\rho_{\mathrm{b}} \mathrm{y} \nabla^{2} \rho_{\mathrm{b}}$ decrecen, reflejando un debilitamiento de estos enlaces como consecuencia a la formación de la nueva interacción $\mathrm{O}$...A $\mathrm{A}$. Es de notar que, a pesar de que los enlaces B-O son covalentes $\nabla^{2} \rho_{h}>0$ en el correspondiente pce, debido a la particular distribución de la densidad electrónica en los enlaces que contiene boro, tal

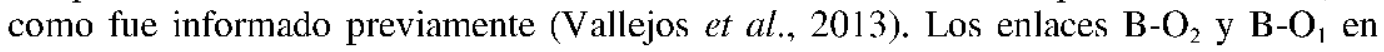
CC-B y CC-C, respectivamente, se acortan y los parámetros topológicos reflejan un ligero fortalecimiento respecto a los enlaces en 1 . Los enlaces $C_{1}-B$ también muestran un pequeño acortamiento y se fortalecen. En los enlaces $C_{1}-C_{2}, C_{2}-C_{3}$, los cambios de los parámetros topológicos son menos significativos o nulos.

En CC-D la interacciónO $\mathrm{O}_{1}{ }^{\cdots} \cdot \mathrm{A} \mathrm{A}$ también muestra características de interacción de capa cerrada, el valor de $\rho_{\mathrm{b}}$ es relativamente bajo, $\nabla^{2} \rho_{\mathrm{b}}>0$ y $\left|V_{\mathrm{b}}\right| / G_{\mathrm{b}}<1$ en el pce $\mathrm{O}_{1}{ }^{\prime} \cdots \mathrm{Al}$. La distancia de enlace de $\mathrm{O}_{1}{ }^{\prime} \cdots \mathrm{Al}\left(1,91 \AA\right.$ y $\rho_{\mathrm{b}}$ en el correspondiente pce $(0,057$ ua.) indican que esta interacción es ligeramente más fuerte que las interacciones entre el átomo de $\mathrm{Al}$ y el $\mathrm{O}$ del boronato, en $\mathbf{C C}-\mathbf{B}$ y $\mathbf{C C}-\mathbf{C}$ en acuerdo con la pequeña diferencia energética que exhiben estos complejos. Como consecuencia de la interacción entre el catalizador y 2 , el enlace $\mathrm{C}_{1}{ }^{\prime}=\mathrm{O}_{1}{ }^{\prime}$ se alarga $(1,22 \AA)$ y $\rho_{\mathrm{b}}$ decrece $(0,378$ ua.) en comparación con 2 ( $d=1,19 \AA$ y $\rho_{\mathrm{b}}=0,419$ ua.).

Estos resultados indican que la interacción entre el catalizador y el reactivo $\mathbf{1}$ ó $\mathbf{2}$, produce un efecto más notable sobre la distribución electrónica de los enlaces cercanos al punto de unión intermolecular.

\section{Estructuras de transición}

En la Fig. 2 se muestran los grafos moleculares de las ETs implicadas en los diferentes caminos de la reacción entre 1 y 2 , con y sin la influencia del catalizador $\mathrm{AlCl}_{3}$ y en la Tabla 3 se expresan las distancias de enlaces y las propiedades topológicas seleccionadas sobre los pces más relevantes para la discusión. 


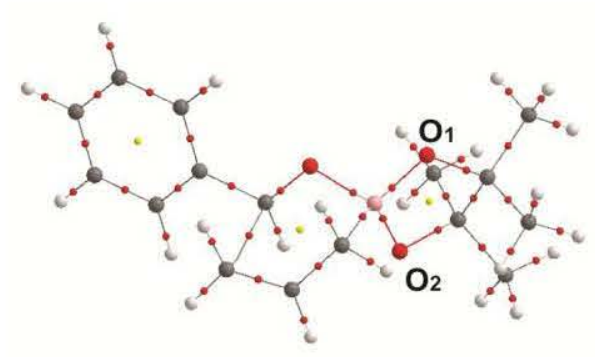

ET-A

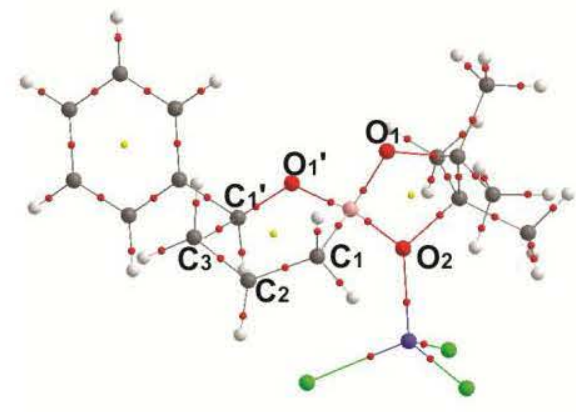

FT-C.

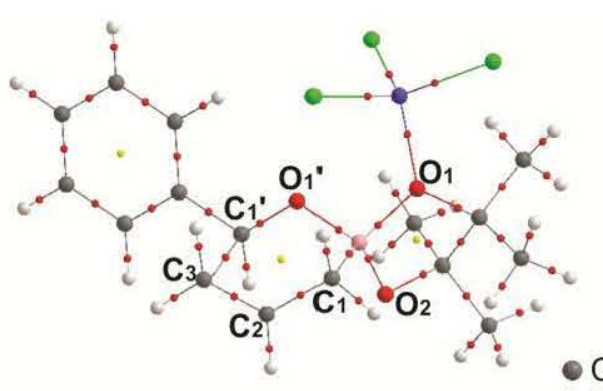

ET-B

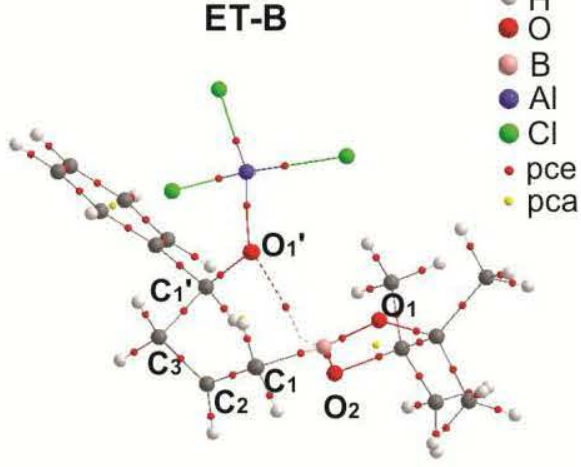

FT-n

Fig. 2: Grafos moleculares para las Ets involucradas en los caminos de la reacción entre 1 y 2 en ausencia (ET-A) y en presencia de $\mathrm{AlCl}_{3}$ (ET-B, ET-C y ET-D).

En los grafos moleculares de las ETs se observa dos pces $\mathrm{B}-\mathrm{O}_{1}{ }^{\prime}$ y $\mathrm{C}_{3}-\mathrm{C}_{1}$, , entre el alilboronato de pinacol y el benzaldehído, asociados con los enlaces en formación. Además, en las ET-(B-D) se observa el pce O...Al de la interacción entre el catalizador y un oxígeno del reactivo.

La distancia B-O ${ }_{1}^{\prime}$ en ET-A es $1,53 \AA$, en ET-B y ET-C son más corta $(1,48 \AA)$ y en ET-D es bastante más larga $(2,45 \AA)$. La interacción B-O' presenta característica de una interacción de capa cerrada (valores de $\rho_{\mathrm{b}}$ relativamente bajos, $\nabla^{2} \rho_{\mathrm{b}}>0$ y $\left|V_{\mathrm{b}}\right| / G_{\mathrm{b}}<1$ ). En el pce $B-\mathrm{O}_{1}{ }^{\prime} \rho_{b}$ toma valores de 0,$125 ; 0,143 ; 0,139$ y 0,022 ua. en ET-A, ET-B, ETC y ET-D, respectivamente, indicando que la interacción está más fortalecida en ET-B y es bastante débil en ET-D. Estos resultados sugieren que en ET-B, y también en ET-C pero en menor medida, el átomo de $\mathrm{B}$ incrementa su carácter electrofílico, como consecuencia de la coordinación del $\mathrm{AlCl}_{3}$ con un átomo de oxígeno del boronato reflejándose en un aumento de la fortaleza del enlace $\mathrm{B}-\mathrm{O}_{1}$ '. 
Tabla 3: Distancias de enlace y propiedades topológicas locales en los pces de los enlaces seleccionados de las ETs bajo estudio.

\begin{tabular}{|c|c|c|c|c|c|}
\hline ETs & interacción & $d$ & $\rho_{b}$ & $\nabla^{2} \rho_{b}$ & $\left|V_{\mathrm{b}}\right| / G_{\mathrm{b}}$ \\
\hline \multirow{6}{*}{ ET-A } & $\mathrm{B}-\mathrm{O}_{1}{ }^{\prime}$ & 1,53 & 0,125 & 0,571 & 1,346 \\
\hline & $\mathrm{C}_{3}-\mathrm{C} 1$, & 2,18 & 0,057 & 0,046 & 1,523 \\
\hline & $\mathrm{B}-\mathrm{C}_{1}$ & 1.70 & 0.132 & $-0,138$ & 2,408 \\
\hline & $\mathrm{B}-\mathrm{O}_{1}$ & 1,41 & 0,185 & 0,767 & 1,428 \\
\hline & $\mathrm{B}-\mathrm{O}_{2}$ & 1,43 & 0,255 & $-0,399$ & 2,405 \\
\hline & $\mathrm{C}_{1}{ }^{\prime}-\mathrm{O}_{1}{ }^{\prime}$ & 1,27 & 0,354 & $-0,041$ & 2,019 \\
\hline \multirow{7}{*}{ ET-B } & $\mathrm{B}-\mathrm{O}_{1}{ }^{\prime}$ & 1,48 & 0,143 & 0,674 & 1,352 \\
\hline & $\mathrm{C}_{3}-\mathrm{C}_{1}$ & 2,11 & 0,066 & 0,038 & 1,641 \\
\hline & $\mathrm{Al} \cdots \mathrm{O}_{1}$ & 1,86 & 0,065 & 0,492 & 0,936 \\
\hline & $\mathrm{B}-\mathrm{C}_{1}{ }^{\mathrm{T}}$ & 1,67 & 0,146 & $-0,192$ & 2,521 \\
\hline & $\mathrm{B}-\mathrm{O}_{1}$ & 1,50 & 0,139 & 0,619 & 1,362 \\
\hline & $\mathrm{B}-\mathrm{O}_{2}$ & 1,40 & 0,188 & 0,773 & 1,434 \\
\hline & $\mathrm{C}_{1}{ }^{\prime}-\mathrm{O}_{1}{ }^{\prime}{ }^{\prime}$ & 1,28 & 0,341 & $-0,057$ & 2,028 \\
\hline \multirow{7}{*}{ ET-C } & $\mathrm{B}-\mathrm{O}_{1}{ }^{\prime}$ & 1,48 & 0,139 & 0,657 & 1,344 \\
\hline & $\mathrm{C}_{3}-\mathrm{C}_{1}$ & 2,10 & 0,068 & 0,038 & 1,654 \\
\hline & $\mathrm{Al} \cdots \mathrm{O}_{2}$ & 1,85 & 0,067 & 0,512 & 0,940 \\
\hline & B-C ${ }_{1}$ & 1,67 & 0,146 & $-0,199$ & 2,549 \\
\hline & $\mathrm{B}-\mathrm{O}_{1}$ & 1,40 & 0,189 & 0,746 & 1,446 \\
\hline & $\mathrm{B}-\mathrm{O}_{2}$ & 1,53 & 0,127 & 0,545 & 1,365 \\
\hline & $\mathrm{C}_{1}{ }^{\prime}-\mathrm{O}_{1}{ }^{\prime}$ & 1,28 & 0,341 & $-0,107$ & 2,053 \\
\hline \multirow{7}{*}{ ET-D } & $\mathrm{B}-\mathrm{O}_{1}{ }^{\prime}$ & 2,45 & 0,022 & 0,056 & 1,059 \\
\hline & $\mathrm{C}_{3}-\mathrm{C}_{1}$ & 1,64 & 0,182 & $-0,291$ & 3,457 \\
\hline & $\mathrm{Al} \cdots \mathrm{O}_{1}$, & 1,79 & 0,077 & 0,627 & 0,947 \\
\hline & $\mathrm{B}-\mathrm{C}_{1}$ & 1,64 & 0,144 & $-0,118$ & 2,276 \\
\hline & $\mathrm{B}-\mathrm{O}_{1}$ & 1,33 & 0,222 & 1,055 & 1,415 \\
\hline & $\mathrm{B}-\mathrm{O}_{2}$ & 1,35 & 0,212 & 0,977 & 1,417 \\
\hline & $\mathrm{C}_{1}{ }^{\prime}-\mathrm{O}_{1}{ }^{\prime}$ & 1,35 & 0,292 & $-0,343$ & 2,251 \\
\hline
\end{tabular}

"Las distancias de enlace $(d)$ se expresan en $\AA$ y los valores de $\rho_{\mathrm{h}} \mathrm{y} \nabla^{2} \rho_{\mathrm{h}}$ en unidades atómicas (ua.). $\left|V_{b}\right| / G_{b}$ es adimensional.

En ET-D la interacción entre el catalizador y $\mathrm{O}_{1}{ }^{\prime}$ produce una disminución en el carácter nucleofílico de $\mathrm{O}_{1}$ ' y por lo tanto la interacción $\mathrm{B}-\mathrm{O}_{1}$ ' es más débil.

Las distanciasde $\mathrm{C}_{3}$ - $\mathrm{C}_{1}{ }^{\prime}$ son más largas en ET-A, ET-B y ET-C $(2,18 ; 2,11 ; 2,10$ $\AA$, respectivamente) que en ET-D $(1,64 \AA)$, además en las primeras ETs los valores de $\rho_{\mathrm{b}}$ son bajos, $\nabla^{2} \rho_{b}>0$ en los pces $C_{3}-C_{1}$, concordando con características de una interacción de capa cerrada, mientras que en ET-D este enlace presenta características de una interacción capa compartida, $\rho_{\mathrm{b}}$ toma valores relativamente altos $\left(0,182\right.$ ua.) y $\nabla^{2} \rho_{\mathrm{b}}$ es $-0,291$ ua. Asimismo, $\left|V_{\mathrm{b}}\right| / G_{\mathrm{b}}$ calculado en el pce $\mathrm{C}_{3}-\mathrm{C}_{1}$ 'para ET-D es 3,457 y en ET-A, ET-B y ET-C toma valores $\sim 1,6$ denotando que en la primera $E T$, para el enlace $C_{3}-C_{1}$ 'el predominio de la densidad de energía potencial sobre la energía cinética es mayor que en las restantes ETs. Estos resultados reflejan que la interacción entre el catalizador y el $\mathrm{O}_{1}{ }$ incrementa el carácter electrofílico de $\mathrm{C}_{1}$ ' favoreciendo la formación del enlace $\mathrm{C}_{3}-\mathrm{C}_{1}$ 'en 
ET-D, respecto a la reacción sin catalizador que ocurre vía ET-A. El efecto que produce la interacción entre el catalizador y un oxígeno del boronato sobre la electrofilicidad del $\mathrm{C}_{1}$ ' es insignificante.

Por otra parte, el enlace Al...O en las ET-B, ET-C y ET-D disminuye la distancia y $\rho_{\mathrm{b}}$ en el pce incrementa, indicando que la interacción entre el catalizador y el reactivo experimenta un ligero fortalecimiento en las ETs respecto a los correspondientes CCs. Además, en las ET-A, ET-B y ET-C los enlaces B-O $\mathrm{O}_{1}$ y B-O $\mathrm{O}_{2}$ aumentan sus distancias y $\rho_{\mathrm{b}}$ en los correspondientes pce disminuye denotando una mayor polarización del enlace, particularmente en ET-B, ET-C, respectivamente mientras en ET-D no se observan cambios significativos en estos enlaces. En la ET-D se produce un significativo alargamiento del enlace $\mathrm{C}_{1}{ }^{\prime}-\mathrm{O}_{1}{ }^{\prime}$ como consecuencia de la evolución de doble a simple enlace, en tanto en las ET-A, ET-B y ET-C el incremento en la distancia de enlace $\mathrm{C}_{1}{ }^{\text {' }} \mathrm{O}_{1}$ ' es menos significativo. Por lo tanto, en las ETs ocurre una redistribución de la densidad electrónicasobre todo el sistema reflejándose en las características de los enlaces, en particular los que se encuentran cercanos al punto de unión con el catalizador.

\section{Carga atómica neta}

$\mathrm{El}$ análisis de las propiedades atómicas integradas se ha empleado en numerosos estudios tanto para comprender como predecir las contribuciones atómicas y de los fragmentos moleculares a determinadas propiedades moleculares (Matta et al., 2007 ${ }^{\mathrm{a}}$; Arabi et al., 2009; Vallejos et al., 2010; Vallejos et al., 2012). La población electrónica de un átomo $N(\Omega)$ se defiende mediante la QTAIM como la integración de la densidad electrónica sobre la cuenca atómica, y puede ser utilizada para calcular la carga neta atómica como $q(\Omega)=N(\Omega)-Z_{\Omega}$, donde $Z_{\Omega}$ es el numero atómico (Bader, 1990). En este trabajo se analizan las $q(\Omega)$ de los átomos seleccionados en los CCs y las ETs, con el propósito de evaluar los cambios que ocurren debido a la interacción catalizador-reactivos 1 y 2 . Se definen cinco fragmentos moleculares: i) BPin, formado por los átomos del heterociclo tetrametildioxaborolano; ii) $\mathrm{C}_{3} \mathrm{H}_{5}$, constituido por los átomos $\mathrm{C}_{1}, \mathrm{C}_{2}$ y $\mathrm{C}_{3}$ y sus átomos de hidrógenos enlazados, iii) $\mathrm{CHO}$, formado por el $\mathrm{O}_{1}{ }^{\prime}, \mathrm{C}_{1}$ ' y el átomos de hidrógeno unido a $\mathrm{C}_{1}{ }^{\prime}$; iv) $\mathrm{C}_{6} \mathrm{H}_{5}$, corresponde a los átomos del grupo fenilo y v) los átomos que conforman el catalizador $\mathrm{AlCl}_{3}$.

En la Tabla 4 se muestran las cargas neta, $q(\Omega)$ delos átomos seleccionados y la sumatoria de las cargas atómicas de los fragmentos moleculares, para los reactivos $\mathbf{1}$ y $\mathbf{2}$, los CCs y las ETs bajo estudio. Merece mencionar que, en todas las especies químicas estudiadas, la sumatoria de la carga neta de todos sus átomos es cero. 
Tabla 4: Cargas netas de los átomos seleccionados y de los fragmentos moleculares (expresadas en unidades atómicas, ua.) calculados en los reactivos, CCs y ETs bajo estudio. a

\begin{tabular}{c|ccccccccc}
\hline átomos & $\mathbf{1}$ & $\mathbf{2}$ & $\mathbf{C C}-\mathbf{B}$ & $\mathbf{C C}-\mathbf{C}$ & $\mathbf{C C}-\mathbf{D}$ & $\mathbf{E T}-\mathbf{A}$ & $\mathbf{E T}-\mathbf{B}$ & $\mathbf{E T}-\mathbf{C}$ & ET-D \\
\hline $\mathrm{C}_{1}$ & $-0,64$ & & $-0,70$ & $-0,69$ & & $-0,41$ & $-0,47$ & $-0,50$ & $-0,51$ \\
$\mathrm{C}_{2}$ & $-0,03$ & & $-0,03$ & $-0,05$ & & $-0,01$ & $-0,01$ & $-0,01$ & 0,00 \\
$\mathrm{C}_{3}$ & $-0,05$ & & $-0,04$ & $-0,04$ & & $-0,10$ & $-0,09$ & $-0,09$ & $-0,02$ \\
$\mathrm{~B}$ & $+2,21$ & & $+2,19$ & $+2,19$ & & $+2,24$ & $+2,23$ & $+2,23$ & 2,23 \\
$\mathrm{O}_{1}$ & $-1,35$ & & $-1,44$ & $-1,35$ & & $-1,35$ & $-1,44$ & $-1,36$ & $-1,35$ \\
$\mathrm{O}_{2}$ & $-1,35$ & & $-1,35$ & $-1,44$ & & $-1,36$ & $-1,37$ & $-1,43$ & $-1,37$ \\
$\mathrm{O}_{1}$ & & $-1,14$ & & & $-1,33$ & $-1,30$ & $-1,35$ & $-1,34$ & $-1,40$ \\
$\mathrm{C}_{1}$ & & $+1,06$ & & & $+0,96$ & $+0,81$ & 0,78 & $+0,77$ & 0,68 \\
$\mathrm{Al}$ & & & $+2,43$ & $+2,44$ & $+2,44$ & & $+2,45$ & $+2,45$ & $+2,44$ \\
Fragmentos & & & & & & & & & \\
\hline $\mathrm{Bpin}$ & $+0,65$ & & $+0,61$ & $+0,60$ & & $+0,63$ & $+0,60$ & $+0,60$ & $+0,68$ \\
$\mathrm{C}_{3} \mathrm{H}_{5}$ & $-0,65$ & & $-0,61$ & $-0,60$ & & $-0,35$ & $-0,31$ & $-0,31$ & $-0,07$ \\
$\mathrm{CHO}$ & & $-0,07$ & & & $-0,30$ & $-0,40$ & $-0,49$ & $-0,46$ & $-0,68$ \\
$\mathrm{Ph}$ & & $+0,07$ & & & $+0,30$ & $+0,12$ & $+0,19$ & $+0,17$ & $+0,07$ \\
\hline
\end{tabular}

${ }^{a} q(\mathrm{Al})$ en el catalizador aislado es $+2,35 \mathrm{e}$. En todas las especies analizadas la suma de las cargas atómicas correspondientes al fragmento $\mathrm{AlCl}_{3}$ es cero. Ver Figuras 1 y 2 para identificar los átomos.

En CC-B y CC-C, se produce una disminución de la carga neta positiva y negativa de los fragmentos $\mathrm{BPin}$ y $\mathrm{C}_{3} \mathrm{H}_{5}$, respectivamente, respecto a los reactivos aislados. En estos $\mathrm{CCs}$ el átomo de $\mathrm{B}$ y el átomo de oxígeno que esta interactuando con el $\mathrm{AlCl}_{3}\left(\mathrm{O}_{1}\right.$ en $\mathbf{C C}-\mathbf{B}$ y $\mathrm{O}_{2}$ en $\mathbf{C C}-\mathbf{C}$ ) incrementan su población electrónica, volviéndose la carga neta de $\mathrm{B}$ menos positiva y la de los oxígenos más negativa. Los átomos $\mathrm{C}_{1}$ en $\mathbf{C C}-\mathbf{B}$ y $\mathbf{C C}-\mathbf{C}$ y también $\mathrm{C}_{2}$ en el último, experimentan un incremento en su población electrónica reflejándose en un aumento de su carga negativa, mientras que $\mathrm{C}_{3}$ en ambos complejos y $\mathrm{C}_{2}$ en CC-B no muestran variaciones significativas en sus cargas netas. Esto sugiere que la carga electrónica que pierde el fragmento $\mathrm{C}_{3} \mathrm{H}_{5}$ se debería a la carga de los átomos de hidrógeno.

En CC-D las cargas negativas/positivas de los fragmentos $\mathrm{CHO} / \mathrm{C}_{6} \mathrm{H}_{5}$ incrementan respecto a 2. En el fragmento $\mathrm{CHO}$ ambos átomos $\mathrm{O}_{1}{ }^{\prime}$ y $\mathrm{C}_{1}{ }^{\prime}$ ganan población electrónica volviéndose su carga neta más negativa y menos positiva, respectivamente.

En los CC-B, CC-C y CC-D se produce un aumento de la carga positiva del Al $(+2,43 ;+2,44$ y $+2,44$ ua., respectivamente $)$ en relación con la carga de este átomo en el catalizador aislado $(+2,35$ ua.). Por lo tanto, como consecuencia de la formación del enlace $\mathrm{Al} \cdots \mathrm{O}$, el $\mathrm{Al}$ dona población electrónica al átomo de oxígeno del reactivo al que se encuentra unido; sin embargo, como en los complejos la carga del fragmento $\mathrm{AlCl}_{3}$ es cero, indica que los átomos de cloro también están ganando población electrónica respecto a lo que ocurre en el catalizador aislado.

Las cargas electrónicas del fragmento BPin en las ET-B y ET-C son menos positiva $(+0,60$ ua.) y en ET-D es más positiva $(+0,68 e)$ que en la ET-A $(+0,63$ ua.) denotando que en las dos primeras ETs la población electrónica es mayor. Es de notar que, lascargas electrónicas del fragmento Bpin en las ET-B y ET-C no muestra variación respecto a los correspondientes $\mathrm{CCs}$, sugiriendo que los pequeños cambios en la pobla- 
ción electrónica de este fragmento se producen como consecuencia de la interacción entre el catalizador y uno de los oxígenos del boronato.

En las cuatro ETs la carga electrónica del átomo de boro es similar $(+2,23)$. Las carga electrónicas de los átomos $\mathrm{O}_{1}$ y $\mathrm{O}_{2}$ en las ET-A y ET-D también son similares y no muestran cambios significativos en relación con el reactivo 1, mientras en las ET-B y ET-C la carga atómica neta del oxígeno enlazado al catalizador es más negativa que en el reactivo 1, pero similar a las cargas calculadas en los correspondientes CCs.

El fragmento $\mathrm{C}_{3} \mathrm{H}_{5}$ exhibe un importante decrecimiento de su carga neta negativa en las ET-B, ET-C y particularmente en la ET-D, respecto a la ET-A, asimismo en comparación con el reactivo 1 en las cuatro ETs se producen una importante disminución de la población electrónica de $\mathrm{C}_{3} \mathrm{H}_{5}$, lo cual puede atribuirse al reordenamiento de la densidad electrónica para la formación del enlace $\mathrm{C}_{3}-\mathrm{C}_{1}$ ' y al cambio de la naturaleza de enlace $\mathrm{C}_{2}-\mathrm{C}_{3}$ (de doble a simple). La carga neta de $\mathrm{C}_{1}$ en las ET-B, ET-C y ET-D $(-0,47$, $-0,50$ y $-0,51 e$, respectivamente) es más negativa que en ET-A. El valor de $q\left(\mathrm{C}_{3}\right)$ es cercano a cero $(-0,02 e)$ en $\mathbf{E T}-\mathbf{D}$, debido a que la formación del enlace $\sigma \mathrm{C}_{3}-\mathrm{C}_{1}$ está más avanzada, en tanto en las ET-B y ET-C la carga neta de $C_{3}$ es similar que en ET-A $(-0,10 e)$.

El fragmento $\mathrm{CHO}$ gana población electrónica incrementando su carga negativa en todas las ETs respecto al reactivo 2, además la carga electrónica negativa de este fragmento es mayor en ET-B, ET-C y principalmente en ET-D que en ET-A. En todos las ETs los átomo $\mathrm{C}_{1}$ ' y $\mathrm{O}_{1}$ ' ganan población electrónica incrementando su carga negativa y disminuyendo su carga positiva, respectivamente. Estas variaciones son más notorias en ET-D.

La carga neta del átomo Al no muestra cambios significativos en las ETs respecto a los correspondientes CCs.

Por otro lado, la sumatoria de las carga de los fragmentos BPin y $\mathrm{C}_{3} \mathrm{H}_{5}$ (correspondiente al fragmento molecular del reactivo alilboronato de pinacol) es de $+0,28,+0,29$, $+0,29$ y +0,63 ua. para ET-A, ET-B, ET-C y ET-D, respectivamente, lo cual refleja la existencia de un flujo de densidad electrónica desde el fragmento del alilboronato de pinacol hacia el fragmento molecular correspondiente al benzaldehído (suma de los fragmentos $\mathrm{CHO}$ y $\mathrm{C}_{6} \mathrm{H}_{5}$ ) en las ETs.

\section{Análisis del Laplaciano de la densidad de carga electrónica}

El análisis topológico de $\nabla^{2} \rho_{\mathrm{r}}$, es una herramienta útil para predecir la posición del sitio para el ataque nucleofílico o electrofílico en sistemas químicos de variada naturaleza (Vallejos et al., 2013; Vallejos et al., 2014b; Carroll et al., 1989; Popelier, 2000b; Zalazar y Peruchena, 2013). La literatura sugiere utilizar la función $L_{\mathrm{r}}=-1 / 4 \nabla^{2} \rho_{\mathrm{r}}$ debido a que intuitivamente se asocia un valor positivo con una zona de concentración de carga y uno negativo con una zona de dilución de carga electrónica, es conveniente. (Popelier, 2000b).

La capa de valencia de un átomo está dividida en una región interna donde $L_{\mathrm{r}}>0$ (llamada concentración de carga en la capa de valencia (CCCV) y una región exterior donde $L_{\mathrm{r}}<0$. Los puntos críticos (pcs) de la función $L_{\mathrm{r}}$ brindan la localización precisa de 
diferentes sitios de reactividad en una molécula. Un pc $(3,-3)$ corresponde a un máximo local en $L_{\mathrm{r}}$ e indica una concentración local de carga electrónica $\left(L_{\mathrm{r}}>0\right)$, además se puede asociar con un máximo enlazante o con máximo no enlazante en la capa de concentración de carga, este último generalmente se localiza donde se esperaría encontrar un par electrónico libre. Los pcs $(3,-1)$ y $(3,+1)$ son puntos de ensilladura dentro de la CCCV (Bader, 1990).

En la Fig. 3 se muestran los mapas de contorno de $L_{\mathrm{r}}$ para los reactivos 1 y 2 y $\operatorname{los}$ complejos CC-(B-D), señalando la posición de los pes en la topología de $L_{\mathrm{r}}$ seleccionados. El análisis se centra sobre los pes de los átomos $\mathrm{B}, \mathrm{C}_{3}, \mathrm{O}_{1}$ ' y $\mathrm{C}_{1}$ ' que participan en la formación de nuevos enlaces en la reacción de alilboración.

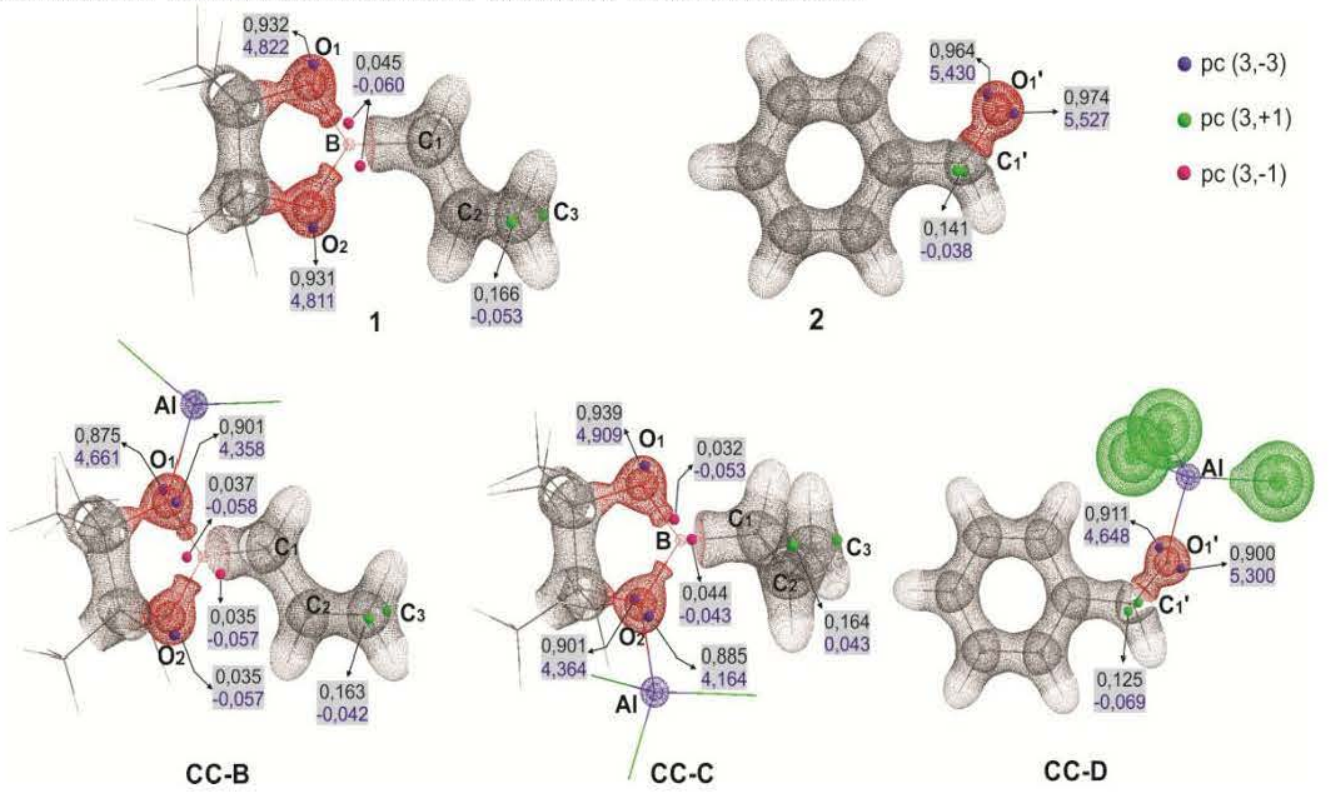

Fig. 3: Envolvente de la función $L_{\mathrm{r}}$ a un contorno externo de 0,00 ua. para los reactivos y CCs bajo estudio, superpuestos a los caminos de enlaces. Se indican los valores de $\delta_{\mathrm{r}}$ y el $L_{\mathrm{r}}$ (en azul) para los pes seleccionados, en ua. Para una mejor visualización, no se muestran las envolventes de algunos átomos.

El átomo $\mathrm{C}_{3}$ en 1, CC-B y CC-C, exhibe en su la capa de valencia dos pcs $(3,+1)$, por encima y por debajo del plano que forman los átomos $\mathrm{H}-\mathrm{C}_{3}-\mathrm{H}$, localizados en zonas de dilución de carga electrónica $\left(L_{\mathrm{r}}<0\right)$. El pc $(3,+1)$ ubicado en el mismo lado que el grupo boronato, puede ser asociado con el sitio de interacción del $\mathrm{C}_{3}$ con el $\mathrm{C}_{1}$ 'del benzaldehído en la reacción alilboración. Los valores de $\rho_{\mathrm{r}}$ y de $L_{\mathrm{r}}$ en el pc $(3,+1)$ del $\mathrm{C}_{3}$ para CC-B y CC-C son similares a los de 1, indicando que la interacción entre el catalizador y el alilboronato no afecta significativamente la reactividad de este centro.

En 1, CC-B y CC-C, también se localizan dos pcs $(3,-1)$, por encima y por debajo del átomo del plano que contiene los enlaces $\mathrm{C}_{1}-\mathrm{B}$ y $\mathrm{B}-\mathrm{O}_{1.2}$. La región deficiente en electrones está relacionada con el orbital p vacante y recientemente se asignó el término "agujero $\pi$ "a la región de baja densidad electrónica situada en dirección perpendicular al centro de una molécula plana o a porción plana de un sistema (Politzer et al., 2013). En 
1, los pcs $(3,-1)$ del átomo de B están equidistantes al núcleo, siendo la distancia de los mismos al núcleo $\left(d_{N}\right)$ de 1,399 ua. y el valor de $\rho_{\mathrm{r}}=0,045$ ua. y $L_{\mathrm{r}}=-0,060$ ua. En CCB el pc $(3,-1)$ que se encuentra del mismo lado que el grupo alilo, que es el sitio que va a interaccionar con el oxígeno carbonílico en la reacción de alilboración, se encuentra más alejado del núcleo $\left(d_{\mathrm{N}}=1,554\right.$ ua.) y presenta menor $\rho_{\mathrm{r}}(0,035$ ua.) que el otro pc $(3,-1)$ $\left(d_{\mathrm{N}}=1,490\right.$ ua. y $\rho_{\mathrm{r}}=0,035$ ua.). En CC-C, se presenta una situación opuesta, el pc $(3,-1)$ que se encuentra en la misma cara que el grupo alilo está más cercano al núcleo $\left(d_{\mathrm{N}}=\right.$ $1,403 \mathrm{au})$ que el otro pc $(3,-1)\left(d_{\mathrm{N}}=1,580 \mathrm{ua}\right)$ y el valor de $\rho_{\mathrm{r}}$ en el primero $\left(\rho_{\mathrm{r}}=0,045\right.$ ua.) es mayor que en el segundo $\left(\rho_{\mathrm{r}}=0,032\right.$ ua.). Estos resultados demuestran que la interacción entre el catalizador y uno de los oxígenos del boronato incrementa la electrofilicidad del átomo de boro respecto al reactivo 1, en ambos complejos CC-B y CC-C, además indican que en $\mathbf{C C}$-B el pc $(3,-1)$ ubicado en la cara en la que se producirá la interacción con el $\mathrm{O}_{1}$ 'es más más susceptible a un ataque nucleofílico que en CC-C, en concordancia con lo reportado en la literatura (Sakata and Fujimoto, 2008).

En el reactivo 2 se encuentra dos pcs máximos enlazante $(3,-3)$ correspondientes a los pares libres del oxígeno carbonílico. Cuando $\mathrm{O}_{1}$ ' interacciona con el $\mathrm{AlCl}_{3}$, en el complejo CC-D, disminuye $\rho_{\mathrm{r}}$ y $L_{\mathrm{r}}$ en los pes $(3,-3)$, en particular en el pc cercano al enlace $\mathrm{Al} \cdots \mathrm{O}_{1}$, , denotando una disminución de su carácter nucleofílico (Popelier et al., 2001). En 2 y CC-D se encuentran dos pcs $(3,+1)$ en la CCVC del $C_{1}$ ' por encima y debajo del plano que contiene al enlace $\mathrm{C}_{1}{ }^{\prime}=\mathrm{O}_{1}{ }^{\prime}$ y yacen en una zona de disminución de carga electrónica $\left(L_{\mathrm{r}}<0\right)$. Las regiones de disminución de carga electrónica $\left(L_{\mathrm{r}}<0\right)$ sobre los átomos de carbono fueron asociadas con sitios de ataques nucleofílicos en metanol y compuestos similares (Carroll et al., 1989; Popelier, 2000a). En CC-D, la densidad electrónica en pc $(3,-1)$ disminuye $\left(\rho_{\mathrm{r}}=1,055\right.$ ua.) y este se aleja de núcleo $\left(d_{\mathrm{N}}=1,055\right.$ ua.) respecto al mismo pc en $2\left(\rho_{\mathrm{r}}=0,141\right.$ ua. y $d_{\mathrm{N}}=1,028$ ua.) reflejando un incremento del carácter electrofílico del $\mathrm{C}_{1}{ }^{\prime}$ como consecuencia de la interacción entre el oxígeno carbonílico y el catalizador.

\section{CONCLUSIONES}

En este trabajo se realizó un análisis de la distribución de la densidad de carga electrónica, en el contexto de la teoría QTAIM, sobre algunas especies involucradas en reacción entre alilboronato de pinacol (1) y benzaldehído (2) en presencia y ausencia de $\mathrm{AlCl}_{3}$ como catalizador. Diferentes parámetros topológicos de la densidad electrónica y de su Laplaciano evaluados en los reactivos, CCs y ETs fueron investigados como potenciales indicadores de reactividad en la reacción de alilboración.

Nuestros resultados demuestran que la interacción entre el catalizador $\mathrm{AlCl}_{3}$ y uno de átomos de oxígeno del reactivo ( 1 ó 2), conlleva a una redistribución de densidad electrónica sobre todo el sistema molecular de los CCs y las ETs, ocurriendo un incremento significativo de la población electrónica en los átomos cercano al sitio de interacción. 
El incremento de la electrofilicidad del átomo de boro en las ETs, en las cuales el $\mathrm{AlCl}_{3}$ se coordina con uno de los átomos de oxígeno del anillo dioxaborolano (ET-B y ET-C), se refleja en un incremento de $\rho_{\mathrm{b}}$ en el pce B-O ${ }_{1}{ }^{\prime}$. En la ET donde el $\mathrm{AlCl}_{3}$ interactúa con el oxígeno del benzaldehído, se produce una disminución en el carácter nucleofílico de $\mathrm{O}_{1}{ }^{\prime}$ denotado por la disminución de $\rho_{\mathrm{b}}$ en el pce B-O,', a su vez en esta ET incrementa el carácter electrofílico de $\mathrm{C}_{1}$ ' expresándose en un aumento de $\rho_{\mathrm{b}}$ en el pce $\mathrm{C}_{3}-\mathrm{C}_{1}$.

El análisis topológico del Laplaciano de la densidad de carga electrónica permitió

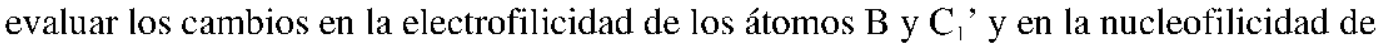
$\mathrm{O}_{1}{ }^{\prime}$ ocurridos como consecuencia de la interacción reactivo-catalizador en los CCs. Además, mediante este análisis, se obtuvo una importante información acerca de los sitios más susceptible para el ataque nucleofílico y electrofílico, relacionados con la reacción de alilboración.

\section{AgRADECIMIENTOS}

Los autores agradecen el apoyo financiero de la Secretaría General de Ciencia y Técnica de la Universidad Nacional del Nordeste (PI-13F005).

Las Dras. Margarita M. Vallejos; María Victoria Avanza y Nélida M. Peruchena son miembros de la Carrera del Investigador del Consejo Nacional de Investigaciones Científicas y Técnicas (CONICET).

\section{BIBLIOGRAFÍA}

ARABI, A.A. y C.F. MATTA, 2009. Where is electronic energy stored in adenosine triphosphate? J. Phys. Chem. A 113: 3360-3368.

BADER, R.F.W., 1990. Atoms in Molecules. A Quantum Theory. London Oxford Science Publications, Clarendon Press.

Blieger-KontG, F., 2000. Calculation of atomic integration data. J. Comput. Chem., 21: 1040-1048.

BRown, E.C.; R.F.W. BADFR y N.H. WtRsTluk, 2009. QTAIM Study on the Degenerate Cope Rearrangements of 1,5-Hexadiene and Semibullvalene. J. Phys. Chem. A 113: 3254-3265.

Brown, H.C.; U.S. RaChtiri a y P.J. Pei.l.e.hiA, 1990. Organoboranes. 53. A high-field variable-temperature proton and boron-11 NMR study of the effects of solvent and structure on reactivity in allylboration. J. Org. Chem., 55: 1868-1874.

Calvo-Losada, S. y J.J. Quirante Sánchez, 2008. Pericyclic versus Pseudopericyclic Reactions. What the Laplacian of the Charge Density, $\nabla 2 \rho(\mathrm{r})$, Has To Say about It? The Case of Cycloaddition Reactions. J. Phys. Chem. A 112: 8164-8178.

Carroll, M.T.; J.R. Cheeseman; R. Osman y H. Weinstein, 1989. Nucleophilic addition to activated double bonds: predictions of reactivity from the Laplacian of the charge density. J. Phys. Chem., 93: 5120-5123.

Corkr, E.J.; C.M. Yu y S.S. Kim, 1989. A practical and efficient method for enantioselective allylation of aldehydes. J. Am. Chem. Soc. 111: 5495-5496. 
Сннск, C.E. у T.M. GIı.внRT', 2005. Progressive Systematic Underestimation of Reaction Energies by the B3LYP Model as the Number of C-C Bonds Increases: Why Organic Chemists Should Use Multiple DFT Models for Calculations Involving Polycarbon Hydrocarbons. J. Org. Chem., 70: 9828-9834.

Denmark, S.E. y J. Fu, 2003. Catalytic Enantioselective Addition of Allylic Organometallic Reagents to Aldehydes and Ketones. Chem. Rev., 103: 2763-2794.

Domingo, L.R.; M.J. AURELl y P. PeRez, 2014. The mechanism of ionic Diels-Alder reactions. A DFT study of the oxa-Povarov reaction. RSC Adv. 4: 16567-16577.

Domingo, L.R.; M.J. Aurell; P. Pérez y R. Contreras, 2002. Quantitative Characterization of the Local Electrophilicity of Organic Molecules. Understanding the Regioselectivity on Diels-Alder Reactions. J. Phys. Chem. A 106: 6871-6875.

Domingio, L.R.; P. Pérez y D.E. ORteg $\Lambda$, 2013. Why Do Five-Membered Heterocyclic Compounds Sometimes Not Participate in Polar Diels-Alder Reactions? J. Org. Chem. 78: 2462-2471.

DOMINGO, L.R.; M.T. PICHER y R.J. ZARAGOZÁ, 1998. Toward an Understanding of the Molecular Mechanism of the Reaction between 1-Methylpyrrole and Dimethyl Acetylenedicarboxylate. An ab Initio Study. J. Org. Chem. 63: 9183-9189.

FRISCH, M.J.T.; H.B. SCHLEGLL; G.E. SCUSERLA; M.A. ROBB; J.R. CHEESEMAN; G. SCALMANI; V. BARONE; B. Mennucci; G.A. Petersson; H. NaKatsuji; M. Caricato; X. Li; H.P. Hratchian; A.F. IzMaylov;

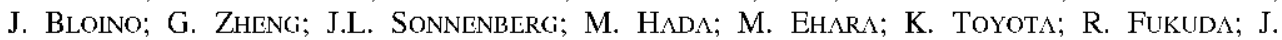
HASEGAW $;$; M. ISHID $\Lambda ;$ T. NAKAJIMA; Y. HOND $;$; O. KITAO; H. NAKA ; T. VREVEN; J.A. MONTGOMERy Jr.; J.E. Peralta; F. Ogliaro; M. BearPark; J.J. Heyd; E. Brothers; K.N. Kudin; V.N. StAROVERov; R. KobAYASHI; J. NORMAND; K. RAGHAVACHARI; A. RENDELL; J.C. BURANT; S.S. Iyengar; J. Tomasi; M. Cossi; N. Rfga; N.J. Mul.am; M. Kı.tne; J.E. Knox; J.B. Cross; V. Bakken; C. Adamo; J. Jaramiloo; R. Gomptíts; R.E. Stratimann; O. Yazyev; A.J. Austin; R. CAMMI; C. POMELLI; J.W. OCHTERSKI; R.L. MARTIN; K. MOROKUMA; V.G. ZAKRZEWSKI; G.A. VOTH; P. Sal.vaidor; J.J. Dannhinberk; S. Dapprich; A.D. Danifls; Ö. Farkas; J.B. Fortesman; J.V. OrTIY; J. Closlowski y D,J, Fox, 2009. Gaussian 09, Revision D.01. Wallingford CT, Gaussian, Inc.

GrIFHTth, G.A.; I.H. HII.IIR; A.C. MORAI.tF; J.M. Pt.RCY; R. ROIG y M.A. VINCENT, 2006. Interplay of Structure and Reactivity in a Most Unusual Furan Diels-Alder Reaction. J. Am. Chem. Soc., 128: 13130-13141.

Gunci, B.W.; X. XuE y W.R. Roush, 2002. The Origin of Diastereofacial Control in Allylboration Reactions Using Tartrate Ester Derived Allylboronates: Attractive Interactions between the Lewis Acid Coordinated Aldehyde Carbonyl Group and an Ester Carbonyl Oxygen. J. Am. Chem. Soc., 124: 10692-10697.

HortmanN. R.W., 1982. Diastereogenic Addition of Crotylmetal Compounds to Aldehydes. Angew. Chem. Int. Ed. 21: 555-566.

Horrmann, R.W. y H.J. ZEIss, 1979. Diastereoselective Synthesis of $\beta$-Methyl Homoallyl Alcohols. Angew. Chem. Int. Ed. Engl. 18: 306-307.

HorfmanN, R.W. y H.J. Ztiss, 1981. Stereoselective synthesis of alcohols. 8. Diastereoselective synthesis of beta-methylhomoallyl alcohols via crotylboronates. J. Org. Chem. 46: 1309-1314.

Ishiy ama, T.; T.A. Ahiко y N. MiyaurA, 2002. Acceleration Effect of Lewis Acid in Allylboration of Aldehydes: Catalytic, Regiospecific, Diastereospecific, and Enantioselective Synthesis of Homoallyl Alcohols†. J. Am. Chem. Soc., 124: 12414-12415.

JoNks, G.O.; V.A. Gunkr y K.N. Houk, 2006. Diels-Alder Reactions of Cyclopentadiene and 9,10Dimethylanthracene with Cyanoalkenes: The Performance of Density Functional Theory and Hartree-Fock Calculations for the Prediction of Substituent Effects. J. Phys. Chem. A, 110: 12161224. 
Ker'Th, T.A., 2011. AIMAll. Overland Park KS, USA, TK Gristmill Software.

KenNedy, J.W.J. y D.G. HaLL, 2002. Dramatic Rate Enhancement with Preservation of Stereospecificity in the First Metal-Catalyzed Additions of Allylboronates. J. Am. Chem. Soc., 124: 11586-1 1587.

KENNEDY, J.W.J. y D.G. HALL, 2003. Recent Advances in the Activation of Boron and Silicon Reagents for Stereocontrolled Allylation Reactions. Angew. Chem. Int. Ed. 42: 4732-4739.

Kennedy, J.W.J. y D.G. HALL, 2004. Lewis Acid Catalyzed Allylboration: Discovery, Optimization, and Application to the Formation of Stereogenic Quaternary Carbon Centers. J. Org. Chem., 69: 44124428.

LACHANCE, H.; X. Lu; M. GRAVEL y D.G. HALL, 2003. Scandium-Catalyzed Allylboration of Aldehydes as a Practical Method for Highly Diastereo- and Enantioselective Construction of Homoallylic Alcohols. J. Am. Chem. Soc., 125: 10160-10161.

LI, Y. y K.N. Houk, 1989. Transition structures for the allylboration reactions of formaldehyde by allylborane and allylboronic acid. J. Am. Chem. Soc., 111: 1236-1240.

LÓPEZ, C.S. y A.R.D. LERA, 2011. Bond Ellipticity as a Measure of Electron Delocalization in Structure and Reactivity. Curr. Org. Chem., 15: 3576-3593.

MATTIA, C.F.; A.A. ARABI y T.A. KHITH, 2007a. Atomic partitioning of the dissociation energy of the P-O(H) bond in hydrogen phosphate anion ( $\mathrm{HPO}_{4}^{2-}$ ): disentangling the effect of $\mathrm{Mg}^{2+}$. J. Phys. Chem. A, 111: 8864-8872.

MATTA, C.F. y R.J. BoYD, 2007b. The Quantum Theory of Atoms in Molecules: from solid state to DNA and drug design. Weinheim, Wiley-VCH.

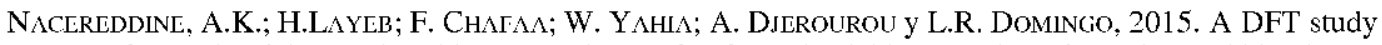
of the role of the Lewis acid catalysts in the $[3+2]$ cycloaddition reaction of the electrophilic nitrone isomer of methyl glyoxylate oxime with nucleophilic cyclopentene. RSC Adv. 5: 64098-64105.

OмоTо, K. y H. Fuııмот, 1998. Theoretical Study of the Effects of Structure and Substituents on Reactivity in Allylboration. The Joumal of Organic Chemistry, 63: 8331-8336.

Politzer, P.; J.S. MuRRAY y T. Clark, 2013. Halogen bonding and other [sigma]-hole interactions: a perspective. Phys. Chem. Chem. Phys., 15: 11178-11189.

Popti.It.R, P.L.A., 2000a. Atoms in Molecules. An Introduction, Harlow, UK, Pearson Education.

POPELIir, P.L.A., 2000b. On the full topology of the Laplacian of the electron density. Coor. Chem. Rev., 197: 169-189.

Popelier, P.L.A. y P.J. Smith, 2001. Protonation energies and the Laplacian of the electron density: A critical examination. Phys. Chem. Chem. Phys., 3: 4208-4212.

RauniYar, V. y D.G. Hal.., 2004. Lewis Acids Catalyze the Addition of Allylboronates to Aldehydes by Electrophilic Activation of the Dioxaborolane in a Closed Transition Structure. J. Am. Chem. Soc., 126: 4518-4519.

Root, J.E. y J.C. Dobrowolsk1, 2007. Variation of BCP ellipticities in the course of the pericyclic and pseudopericyclic $[2 \& \# x a 0 ;+\& \# x a 0 ; 2]$ cycloaddition reactions of cumulenes. Chem. Phys.Lett., 449: 240-245.

SaKatA, K. y H. FujtMoto, 2008. Quantum Chemical Study of Lewis Acid Catalyzed Allylboration of Aldehydes. J. Am. Chem. Soc., 130: 12519-12526.

Villejos, M.M.; E.L. Angielina y N.M. Peruchena, 2010. Bifunctional Hydrogen Bonds in Monohydrated Cycloether Complexes. J. Phys. Chem. A, 114: 2855-2863. 
Vali.fos, M.M.; N. Grimblat y S.C. Phil.fgrinet, 2014a. Diels-Alder reactions of pinacol alkenylboronates: an experimental and theoretical study. RSC Adv. 4: 36385-36400.

VALI.jos, M.M.; N. GRIMBi.AT y S.C. Phi.I.FGRINET, 2014b. Reactivity and Selectivity of Boron-Substituted Alkenes in the Diels-Alder Reaction with Cyclopentadiene. A Study of the Electron Charge Density and Its Laplacian. J. Phys. Chem. A, 118: 5559-5570.

Vallejos, M.M. y S.C. Pellegrinet, 2015. Competing mechanisms for the reaction of dichloropropynylborane with 2-tert-butylbutadiene. Diels-Alder reaction versus alkynylboration. RSC Advances 5: 70147-70155.

Vallejos, M.M. y N.M. Peruchena, 2012. Preferential Formation of the Different Hydrogen Bonds and Their Effects in Tetrahydrofuran and Tetrahydropyran Microhydrated Complexes. J. Phys. Chem. A, 116: 4199-4210.

Vali.fjos, M.M.; N.M. Ptruchena y S.C. PhLI.FGRINeT, 2013. [4 + 3] and [4 + 2] mechanisms of the DielsAlder reactions of vinylboranes: an analysis of the electron charge density distribution. Org. Biomol. Chem., 11: 7953-7965.

WAGNER, G.; T.N. DANKS y V. Vullo, 2007. Quantum-chemical study of the Lewis acid influence on the cycloaddition of benzonitrile oxide to acetonitrile, propyne and propene. Tetrahedron, 63: 52515260 .

WERSTIUK, N.H. y W. SOKOL, 2008. QTAIM-DI-VISAB computational study on the Diels-Alder reaction of cyclopentadiene. On the nature of the so-called secondary orbital interactions. Can. J. Chem., 86: $737-744$.

YAMAMO'TO, Y. y N. ASAO, 1993. Selective reactions using allylic metals. Chem. Rev, 93: 2207-2293.

ZALAZAR, M. y N. Peruchena 2011. Topological description of the bond-breaking and bond-forming processes of the alkene protonation reaction in zeolite chemistry: an AIM study. J. Mol. Model., 17: 2501-2511.

ZAI.AZAR, M.F. y N.M. PFiruchina, 2013. Laplacian of the electron density: a hole-lump interaction as a tool to study stereoelectronic control of chemical reactions. J. Phys. Org. Chem., 27: 327-335.

ZHaO, Y. y D.G. TRuHLAR, 2004. Hybrid Meta Density Functional Theory Methods for Thermochemistry, Thermochemical Kinetics, and Noncovalent Interactions: The MPW1B95 and MPWB1K Models and Comparative Assessments for Hydrogen Bonding and van der Waals Interactions. J. Phys. Chem. A, 108: 6908-6918. 